\title{
NON-PERIODIC GEODESIC BALL PACKINGS GENERATED BY INFINITE REGULAR PRISM TILINGS IN $\mathrm{SL}_{2} \mathrm{R}$ SPACE
}

\author{
JENŐ SZIRMAI
}

\begin{abstract}
In [14] we defined and described the regular infinite or bounded $p$-gonal prism tilings in $\widetilde{\mathbf{S L}_{2} \mathbf{R}}$ space. We proved that there exist infinitely many regular infinite $p$-gonal face-to-face prism tilings $\mathcal{T}_{p}^{i}(q)$ and infinitely many regular bounded $p$-gonal non-face-to-face prism tilings $\mathcal{T}_{p}(q)$ for integer parameters $p, q, 3 \leq p, 2 p /(p-2)<q$. Moreover, in $[\mathbf{5}, \mathbf{7}]$ we have determined the symmetry group of $\mathcal{T}_{p}(q)$ via its index 2 rotational subgroup, denoted by $\mathbf{p q} \mathbf{2}_{\mathbf{1}}$ and investigated the corresponding geodesic and translation ball packings.

In this paper, we study the structure of the regular infinite or bounded $p$-gonal prism tilings and we prove that the side curves of their base figures are arcs of Euclidean circles for each parameter. Furthermore, we examine the non-periodic geodesic ball packings of congruent regular nonperiodic prism tilings derived from the regular infinite $p$ gonal face-to-face prism tilings $\mathcal{T}_{p}^{i}(q)$ in $\widetilde{\mathbf{S L}_{2} \mathbf{R}}$ geometry. We develop a procedure to determine the densities of the above non-periodic optimal geodesic ball packings and apply this algorithm to them. We search for values of parameters $p$ and $q$ that provide the largest packing density. In this paper, we obtain greater density $0.626606 \ldots$ for $(p, q)=(29,3)$ than the maximum density of the corresponding periodic geodesic ball packings under the groups $\mathbf{p q} \mathbf{2}_{\mathbf{1}}$.

In our work we use the projective model of $\widetilde{\mathbf{S L}_{2} \mathbf{R}}$ introduced by Molnár in [2].
\end{abstract}

1. Basic notions. The real $2 \times 2$ matrices $\left(\begin{array}{ll}d & b \\ c & a\end{array}\right)$ with unit determinant $a d-b c=1$ constitute a Lie transformation group by the usual product operation, taken to act on row matrices as on point coordinates

2010 AMS Mathematics subject classification. Primary 51M20, 52B15, 52C17, $52 \mathrm{C} 22,53 \mathrm{~A} 35$.

Keywords and phrases. Finite fields, permutation polynomials, HermiteDickson's theorem.

Received by the editors on March 13, 2014, and in revised form on August 9, 2014. 
on the right as follows:

$$
\begin{gathered}
\left(z^{0}, z^{1}\right)\left(\begin{array}{ll}
d & b \\
c & a
\end{array}\right)=\left(z^{0} d+z^{1} c, z^{0} b+z^{1} a\right)=\left(w^{0}, w^{1}\right) \\
\text { with } \quad w=\frac{w^{1}}{w^{0}}=\frac{b+\left(z^{1} / z^{0}\right) a}{d+\left(z^{1} / z^{0}\right) c}=\frac{b+z a}{d+z c},
\end{gathered}
$$

as action on the complex projective line $\mathbf{C}^{\infty}$ (see $\left.[\mathbf{2}, \mathbf{3}]\right)$. This group is a three-dimensional manifold, because of its three independent real coordinates and with its usual neighborhood topology $([\mathbf{8}, \mathbf{9}, \mathbf{1 6}])$. In order to model the above structure in the projective sphere $\mathcal{P S}^{3}$ and in the projective space $\mathcal{P}^{3}$ (see [2]), we introduce the new projective coordinates $\left(x^{0}, x^{1}, x^{2}, x^{3}\right)$ where

$$
a:=x^{0}+x^{3}, \quad b:=x^{1}+x^{2}, \quad c:=-x^{1}+x^{2}, \quad d:=x^{0}-x^{3},
$$

with the positive, then the non-zero multiplicative equivalence as projective freedom in $\mathcal{P S}^{3}$ and in $\mathcal{P}^{3}$, respectively. Then it follows that $0>b c-a d=-x^{0} x^{0}-x^{1} x^{1}+x^{2} x^{2}+x^{3} x^{3}$ describes the interior of the above one-sheeted hyperboloid solid $\mathcal{H}$ in the usual Euclidean coordinate simplex with the origin $E_{0}(1 ; 0 ; 0 ; 0)$ and the ideal points of the axes

$$
E_{1}^{\infty}(0 ; 1 ; 0 ; 0), \quad E_{2}^{\infty}(0 ; 0 ; 1 ; 0), \quad E_{3}^{\infty}(0 ; 0 ; 0 ; 1) .
$$

We consider the collineation group $\mathbf{G}_{*}$ that acts on the projective sphere $\mathcal{S P}^{3}$ and preserves a polarity, i.e., a scalar product of signature $(--++)$, this group leaves the one sheeted hyperboloid solid $\mathcal{H}$ invariant. We have to choose an appropriate subgroup $\mathbf{G}$ of $\mathbf{G}_{*}$ as an isometry group. Then the universal covering group and space $\widetilde{\mathcal{H}}$ of $\mathcal{H}$ will be the hyperboloid model of $\widetilde{\mathbf{S L}_{2} \mathbf{R}},[2]$.

The specific isometries $\mathbf{S}(\phi), \phi \in \mathbf{R}$, constitute a one parameter group given by the matrices:

$$
\mathbf{S}(\phi):\left(s_{i}^{j}(\phi)\right)=\left(\begin{array}{cccc}
\cos \phi & \sin \phi & 0 & 0 \\
-\sin \phi & \cos \phi & 0 & 0 \\
0 & 0 & \cos \phi & -\sin \phi \\
0 & 0 & \sin \phi & \cos \phi
\end{array}\right) .
$$

The elements of $\mathbf{S}(\phi)$ are the so-called fibre translations. We obtain a unique fibre line to each $X\left(x^{0} ; x^{1} ; x^{2} ; x^{3}\right) \in \widetilde{\mathcal{H}}$ as the orbit under the right action of $\mathbf{S}(\phi)$ on $X$. The coordinates of points lying on the fibre 
line through $X$ can be expressed as the images of $X$ by $\mathbf{S}(\phi)$ :

$$
\begin{gathered}
\left(x^{0} ; x^{1} ; x^{2} ; x^{3}\right) \stackrel{\mathbf{S}(\phi)}{\longrightarrow}\left(x^{0} \cos \phi-x^{1} \sin \phi ; x^{0} \sin \phi+x^{1} \cos \phi ;\right. \\
\left.x^{2} \cos \phi+x^{3} \sin \phi ;-x^{2} \sin \phi+x^{3} \cos \phi\right) .
\end{gathered}
$$

The points of a fibre line through $X$ by usual inhomogeneous Euclidean coordinates

$$
x=\frac{x^{1}}{x^{0}}, \quad y=\frac{x^{2}}{x^{0}}, \quad z=\frac{x^{3}}{x^{0}}, \quad x^{0} \neq 0
$$

are given by

$$
(1 ; x ; y ; z) \stackrel{\mathbf{s}(\phi)}{\longrightarrow}\left(1 ; \frac{x+\tan \phi}{1-x \tan \phi} ; \frac{y+z \tan \phi}{1-x \tan \phi} ; \frac{z-y \tan \phi}{1-x \tan \phi}\right)
$$

for the projective space $\mathcal{P}^{3}$, where ideal points (at infinity) conventionally occur.

In (1.3) and (1.4) we can see the $2 \pi$ periodicity of $\phi$, moreover the (logical) extension to $\phi \in \mathbf{R}$, as a real parameter, to have the universal covers $\widetilde{\mathcal{H}}$ and $\widehat{\mathbf{S L}_{2} \mathbf{R}}$, respectively, through the projective sphere $\mathcal{P S}^{3}$. The elements of the isometry group of $\mathbf{S L}_{\mathbf{2}} \mathbf{R}$ (and so by the above extension the isometries of $\left.\widehat{\mathbf{S L}_{2} \mathbf{R}}\right)$ can be described by the matrix $\left(a_{i}^{j}\right)$, see $[\mathbf{2}, \mathbf{3}]$. Moreover, we have the projective proportionality, of course. We define the translation group $\mathbf{G}_{T}$ as a subgroup of the isometry group of $\mathbf{S L}_{\mathbf{2}} \mathbf{R}$, the isometries acting transitively on the points of $\mathcal{H}$ and by the above extension on the points of $\widehat{\mathbf{S L}_{2} \mathbf{R}}$ and $\widetilde{\mathcal{H}} . \mathbf{G}_{T}$ maps the origin $E_{0}(1 ; 0 ; 0 ; 0)$ onto $X\left(x^{0} ; x^{1} ; x^{2} ; x^{3}\right)$. These isometries and their inverses (up to a positive determinant factor) are given by the following matrices:

$$
\mathbf{T}:\left(t_{i}^{j}\right)=\left(\begin{array}{cccc}
x^{0} & x^{1} & x^{2} & x^{3} \\
-x^{1} & x^{0} & x^{3} & -x^{2} \\
x^{2} & x^{3} & x^{0} & x^{1} \\
x^{3} & -x^{2} & -x^{1} & x^{0}
\end{array}\right)
$$

The rotation about the fibre line through the origin $E_{0}(1 ; 0 ; 0 ; 0)$ by angle $\omega(-\pi<\omega \leq \pi)$ can be expressed by the following matrix, 
see $[2]$

$$
\mathbf{R}_{E_{0}}(\omega):\left(r_{i}^{j}\left(E_{0}, \omega\right)\right)=\left(\begin{array}{cccc}
1 & 0 & 0 & 0 \\
0 & 1 & 0 & 0 \\
0 & 0 & \cos \omega & \sin \omega \\
0 & 0 & -\sin \omega & \cos \omega
\end{array}\right)
$$

and the rotation $\mathbf{R}_{X}(\omega)$ about the fibre line through $X\left(x^{0} ; x^{1} ; x^{2} ; x^{3}\right)$ by angle $\omega$ can be derived by formulas (1.5) and (1.6):

$$
\mathbf{R}_{X}(\omega)=\mathbf{T}^{-1} \mathbf{R}_{E_{0}}(\omega) \mathbf{T}:\left(r_{i}^{j}(X, \omega)\right) .
$$

Horizontal intersection of the hyperboloid solid $\mathcal{H}$ with the plane $E_{0} E_{2}^{\infty} E_{3}^{\infty}$ provides the hyperbolic $\mathbf{H}^{2}$ base plane of the model $\widetilde{\mathcal{H}}=$ $\widehat{\mathbf{S L}_{2} \mathbf{R}}$. The fibre through $X$ intersects the base plane $z^{1}=x=0$ in the foot point

$$
Z\left(z^{0}=x^{0} x^{0}+x^{1} x^{1} ; z^{1}=0 ; z^{2}=x^{0} x^{2}-x^{1} x^{3} ; z^{3}=x^{0} x^{3}+x^{1} x^{2}\right) .
$$

After [2], we introduce the so-called hyperboloid parametrization as follows:

$$
\begin{array}{rlrl} 
& x^{0}=\cosh r \cos \phi, & x^{1}=\cosh r \sin \phi, \\
x^{2}=\sinh r \cos (\theta-\phi), & x^{3}=\sinh r \sin (\theta-\phi),
\end{array}
$$

where $(r, \theta)$ are polar coordinates of the base plane and $\phi$ is just the fibre coordinate. We note that

$$
-x^{0} x^{0}-x^{1} x^{1}+x^{2} x^{2}+x^{3} x^{3}=-\cosh ^{2} r+\sinh ^{2} r=-1<0 .
$$

The inhomogeneous coordinates corresponding to (1.9), that play an important role in the later visualization of prism tilings in $\mathbf{E}^{3}$, are given by

$$
\begin{gathered}
x=\frac{x^{1}}{x^{0}}=\tan \phi, \quad y=\frac{x^{2}}{x^{0}}=\tanh r \frac{\cos (\theta-\phi)}{\cos \phi}, \\
z=\frac{x^{3}}{x^{0}}=\tanh r \frac{\sin (\theta-\phi)}{\cos \phi} .
\end{gathered}
$$




\subsection{Geodesic balls in $\widetilde{\mathrm{SL}_{2} \mathrm{R}}$.}

Definition 1.1. The distance $d\left(P_{1}, P_{2}\right)$ between the points $P_{1}$ and $P_{2}$ is defined by the arc length of the geodesic curve from $P_{1}$ to $P_{2}$.

Definition 1.2. The geodesic sphere of radius $\rho$ (denoted by $S_{P_{1}}(\rho)$ ) with the center at $P_{1}$ is defined as the set of all points $P_{2}$ satisfying $d\left(P_{1}, P_{2}\right)=\rho$. Moreover, we require that the geodesic sphere is a simply connected surface without self-intersection.

Definition 1.3. The body of the geodesic sphere of center $P_{1}$ and with radius $\rho$ is called geodesic ball, denoted by $B_{P_{1}}(\rho)$, i.e., $Q \in B_{P_{1}}(\rho)$ if and only if $0 \leq d\left(P_{1}, Q\right) \leq \rho$.

From the results [5] it follows that if $\rho \in\left[0, \frac{\pi}{2}\right)$ then $S(\rho)$ is a simply connected surface in $\mathbf{E}^{3}$ and $\widetilde{\mathbf{S L}_{2} \mathbf{R}}$, respectively. If $\rho \geq \frac{\pi}{2}$ then the universal cover should be discussed. Therefore, we consider geodesic spheres and balls only with radii $\rho \in\left[0, \frac{\pi}{2}\right)$ in what follows.

1.2. The volume of a geodesic ball. The volume formula of the geodesic ball $B(\rho)$ follows from the metric tensor $g_{i j}$, see [5]. We obtain the connection between the hyperboloid coordinates $(r, \theta, \phi)$ and the geographical coordinates $(s, \lambda, \alpha)$ in a standard way. Therefore, the volume of the geodesic ball of radius $\rho$ can be computed by the following:

\section{Theorem 1.4.}

$$
\begin{aligned}
\operatorname{Vol}(B(\rho))= & \int_{B} \frac{1}{2} \sinh (2 r) d r d \theta d \phi \\
= & 4 \pi \int_{0}^{\rho} \int_{0}^{\pi / 4} \frac{1}{2} \sinh (2 r(s, \alpha)) \dot{j} J_{1} \mid d \alpha d s \\
& +4 \pi \int_{0}^{\rho} \int_{\pi / 4}^{\pi / 2} \frac{1}{2} \sinh (2 r(s, \alpha)) \dot{\mid} J_{2} \mid d \alpha d s,
\end{aligned}
$$

where $\left|J_{1}\right|=\left|\begin{array}{ll}\partial r / \partial s & \partial r / \partial \alpha \\ \partial \phi / \partial s & \partial \phi / \partial \alpha\end{array}\right|$ and similarly $\left|J_{2}\right|($ by Table 1 and $\partial \theta / \partial \lambda=1)$ are the corresponding Jacobians. 


\subsection{Regular bounded periodic prism tilings and their space} groups $\mathbf{p q} 2_{1}$. In [14], we defined and described the regular prisms and prism tilings with a space group class $\Gamma=\mathbf{p q} \mathbf{2}_{\mathbf{1}}$ of $\widetilde{\mathbf{S L}_{2} \mathbf{R}}$. These will be summarized in this section.

Definition 1.5. Let $\mathcal{P}^{i}$ be an infinite solid that is bounded by certain surfaces determined (as in [14]) by "side fibre lines" passing through the vertices of a regular $p$-gon $\mathcal{P}^{b}$ lying in the base plane. The images of solids $\mathcal{P}^{i}$ by $\widetilde{\mathbf{S L}_{2} \mathbf{R}}$ isometries are called infinite regular p-sided prisms. Here, regular means that the side surfaces are congruent to each other under rotations about a fiber line (e.g., through the origin).

The common part of $\mathcal{P}^{i}$, with the base plane is the base figure of $\mathcal{P}^{i}$ that is denoted by $\mathcal{P}$ and its vertices coincide with the vertices of $\mathcal{P}^{b}$, but $\mathcal{P}$ is not assumed to be a polygon.

Definition 1.6. A bounded regular p-sided prism is an isometric image of a solid which is bounded by the side surfaces of a regular $p$-sided infinite prism $\mathcal{P}^{i}$, its base figure $\mathcal{P}$ and the translated copy $\mathcal{P}^{t}$ of $\mathcal{P}$ by a fibre translation, given by (1.2). The faces $\mathcal{P}$ and $\mathcal{P}^{t}$ are called cover faces.

We consider regular prism tilings $\mathcal{T}_{p}(q)$ by prisms $\mathcal{P}_{p}(q)$ where $q$ pieces regularly meet at each side edge by $q$-rotation.

The following theorem has been proved in [14].

Theorem 1.7. There exist regular, bounded prism tilings $\mathcal{T}_{p}(q)$ in $\widetilde{\mathbf{S L}_{2} \mathbf{R}}$ that are not face-to-face for all integers $p$ and $q$ such that $3 \leq p$ and $2 p /(p-2)<q$.

We assume that the prism $\mathcal{P}_{p}(q)$ is a topological polyhedron having at each vertex one $p$-gonal cover face (it is not a polygon at all) and two skew quadrangles which lie on certain side surfaces in the model. Let $\mathcal{P}_{p}(q)$ be one of the tiles of $\mathcal{T}_{p}(q) ; \mathcal{P}^{b}$ is centered in the origin with vertices $A_{1} A_{2} A_{3} \cdots A_{p}$ in the base plane (Figures 1 and 2). It is clear that the side curves, $c_{A_{i} A_{i+1}}, i=1, \ldots, p, A_{p+1} \equiv A_{1}$, of the base figure are derived from each other by $2 \pi / p$ rotation about the vertical 
$x$ axis, so they are congruent in the $\widetilde{\mathbf{S L}_{2} \mathbf{R}}$ sense. The corresponding vertices $B_{1} B_{2} B_{3} \cdots B_{p}$ are generated by a fibre translation $\tau$ given by (1.3) with real parameter $\Phi>0$. The fibre lines through the vertices $A_{i} B_{i}$ are denoted by $f_{i}, i=1, \ldots, p$, and the fibre line through the "midpoint" $H$ of the curve $c_{A_{1} A_{p}}$ is denoted by $f_{0}$. This $f_{0}$ will be a half-screw axis as follows below.

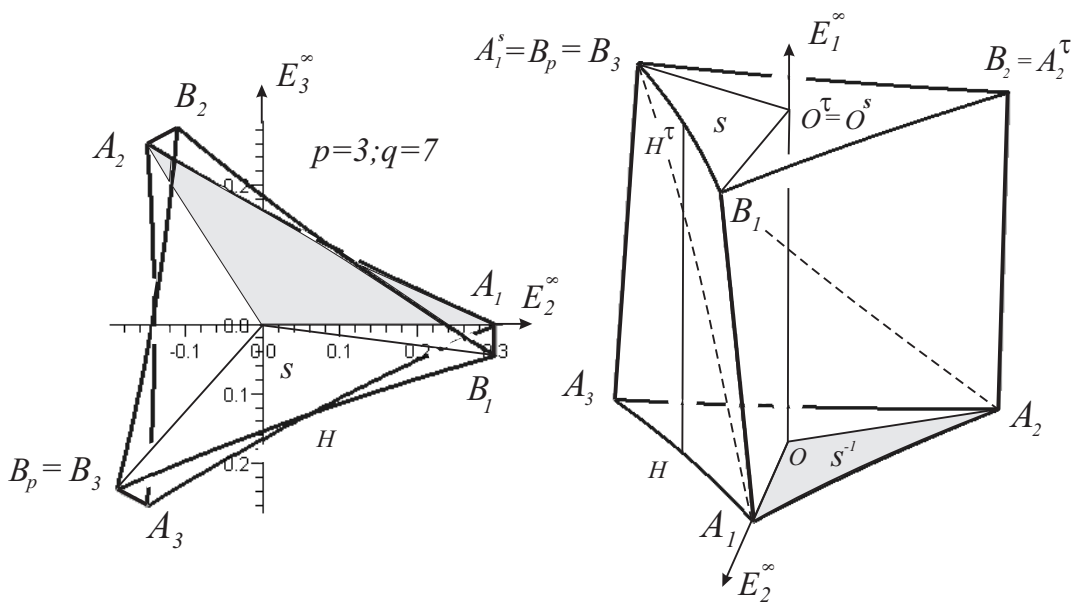

FiguRE 1. The regular prism $\mathcal{P}_{p}(q)$ and the fundamental domain of the space group pq2 1

The tiling $\mathcal{T}_{p}(q)$ is generated by a discrete isometry group $\Gamma_{p}(q)=$ pq2 $1 \subset$ Isom $\left(\widetilde{\mathbf{S L}_{2} \mathbf{R}}\right)$ given by its fundamental domain $A_{1} A_{2} O A_{1}^{\mathbf{s}} A_{2}^{\mathbf{s}} O^{\mathbf{s}}$ a topological polyhedron and the group presentation (see Figures 1 and 4 for $p=3$ and [14] for details):

$$
\begin{aligned}
\mathbf{p q} \mathbf{2}_{\mathbf{1}} & =\left\{\mathbf{a}, \mathbf{b}, \mathbf{s}: \mathbf{a}^{p}=\mathbf{b}^{q}=\mathbf{a s a}^{-1} \mathbf{s}^{-1}=\mathbf{b a b s}^{-1}=\mathbf{1}\right\} \\
& =\left\{\mathbf{a}, \mathbf{b}: \mathbf{a}^{p}=\mathbf{b}^{q}=\mathbf{a b a b a} \mathbf{b}^{-1} \mathbf{b}^{-1} \mathbf{a}^{-1} \mathbf{b}^{-1}=\mathbf{1}\right\} .
\end{aligned}
$$

Here $\mathbf{a}$ is a $p$-rotation about the fibre line through the origin ( $x$ axis), $\mathbf{b}$ is a $q$-rotation about the fibre line trough $A_{1}$ and $\mathbf{s}=\mathbf{b a b}$ is a screw motion s : $O A_{1} A_{2} \rightarrow O^{\mathbf{s}} B_{p} B_{1}$. All these can be obtained by formulas (1.5) and (1.6). Then we get that $\mathbf{a b a b}=\mathbf{b a b a}=: \tau$ is a fibre translation. Then $\mathbf{a b}$ is a $\mathbf{2}_{\mathbf{1}}$ half-screw motion about $f_{0}=H H^{\tau}$ (see Figure 1) that also determines the fibre translation $\tau$ above. This 
group in (3.1) surprisingly occurred in [6, Section 6] at double links $K_{p, q}$. The coordinates of the vertices $A_{1} A_{2} A_{3} \cdots A_{p}$ of the base figure and the corresponding vertices $B_{1} B_{2} B_{3} \cdots B_{p}$ of the cover face can be computed for all given parameters $p, q$ by

$$
\tanh \left(O A_{1}\right)=b:=\sqrt{\frac{1-\tan \pi / p \tan \pi / q}{1+\tan \pi / q \tan \pi / q}} .
$$

1.4. The volume of the bounded regular prisms. The volume formula of a sector-like three dimensional domain $\operatorname{Vol}(D(\Psi))$ can be computed routinely by the metric tensor $g_{i j}$, see [5], in the hyperboloid coordinates. This is defined by the base figure $D$ lying in the base plane and by fibre translation $\tau$ given by (1.3) with the height parameter $\Psi$.

Theorem 1.8. Suppose we are given a sector-like region $D$, a continuous function $r=r(\theta)$, where the radius $r$ depends upon the polar angle $\theta$. The volume of the domain $D(\Psi)$ ) is derived by the following integral:

$$
\begin{aligned}
\operatorname{Vol}(D(\Psi)) & =\int_{D} \frac{1}{2} \sinh (2 r(\theta)) /, \mathrm{d} r \mathrm{~d} \theta \mathrm{d} \psi \\
& =\int_{0}^{\Psi} \int_{\theta_{1}}^{\theta_{2}} \int_{0}^{r(\theta)} \frac{1}{2} \sinh (2 r(\theta)) \mathrm{d} r \mathrm{~d} \theta \mathrm{d} \psi \\
& =\Psi \int_{\theta_{1}}^{\theta_{2}} \frac{1}{4}(\cosh (2 r(\theta))-1) \mathrm{d} \theta .
\end{aligned}
$$

Letting $\mathcal{P}_{p}(q)$ be an arbitrary bounded regular prism, we get the following.

Theorem 1.9. The volume of the bounded regular prism $\mathcal{P}_{p}(q)(3 \leq$ $p \in \mathbb{N}, 2 p /(p-2)<q \in \mathbb{N})$ is given by the following simple formula:

$$
\operatorname{Vol}\left(\mathcal{P}_{p}(q)\right)=\operatorname{Vol}(D(p, q, \Psi)) \cdot p,
$$

where $\operatorname{Vol}(D(p, q, \Psi))$ is the volume of the sector-like three dimensional domain given by the sector region $O A_{1} A_{2} \subset \mathcal{P}$ (see Figures 1 and 3 ) and by $\Psi$, the $\widetilde{\mathbf{S L}_{2} \mathbf{R}}$ height of the prism, depending on $p, q$. 

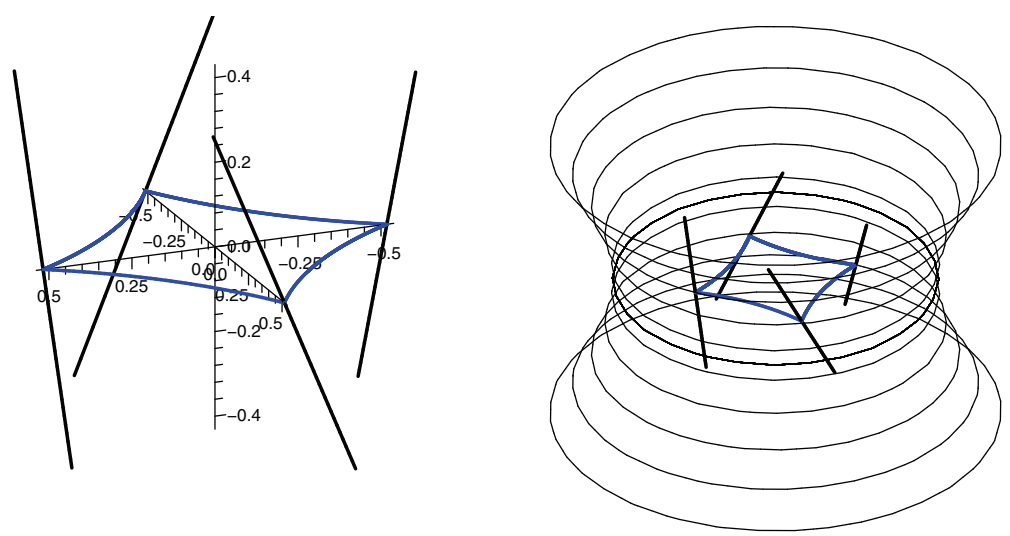

FIGURE 2. Regular infinite 4-gonal prism $\mathcal{P}_{4}^{i}(6)$ of the infinite regular prism tiling $\mathcal{T}_{4}^{i}(6)$.

\section{Regular infinite prism tilings and non-periodic ball pack-} ings.

2.1. Infinite regular prism tilings. In this subsection, we study the regular infinite prism tilings $\mathcal{T}_{p}^{i}(q)$. Let $\mathcal{T}_{p}(q)$ be a regular prism tiling, and let $\mathcal{P}_{p}(q)$ be one of its tiles given by its base figure $\mathcal{P}$, centered at the origin $K$ with vertices $G_{1} G_{2} G_{3} \cdots G_{p}$ in the base plane of the model and the corresponding vertices $A_{1} A_{2} A_{3} \cdots A_{p}$ and $B_{1} B_{2} B_{3} \cdots B_{p}$ generated by fibre translations $-\tau$ and $\tau$ given by (1.3) with parameter $\Psi=\pi / 2-\pi / p-\pi / q$. The images of the topological polyhedron $\mathcal{P}_{p}(q)$ by the translations $\langle\tau\rangle$ form an infinite prism $\mathcal{P}_{p}^{i}(q)$ (see Definitions 1.5 and 1.6).

By the construction of the bounded prism tilings it follows that the rotation through $\omega=2 \pi / q$ about the fibre lines $f_{i}$ maps the corresponding side face onto the neighboring one. Therefore, we obtain the following (see [14]):

Theorem 2.1. There exist regular infinite face-to-face prism tilings $\mathcal{T}_{p}^{i}(q)$ for integer parameters $p \geq 3$ and $q>2 p /(p-2)$. 

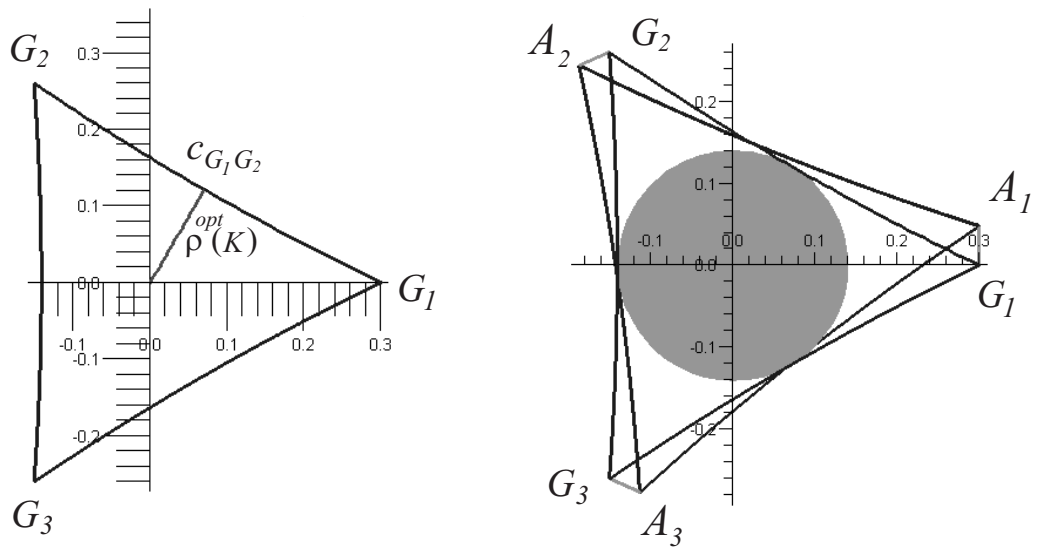

FiguRE 3. The maximum radius $\rho^{\text {opt }}(K)$ and the optimal half-prism $A_{1} A_{2} A_{3} G_{1} G_{2} G_{3}$ with the optimal half-sphere for parameters $p=3, q=7$ with the maximum radius

For example, we have described $\mathcal{P}_{4}^{i}(6)$ with its base polygon in Figure 2 , with $b=(\sqrt{6}-\sqrt{2}) / 2$.

2.2. Non-periodic geodesic ball packings. We consider an infinite regular prism tiling $\mathcal{T}_{p}^{i}(q)$ and let $\mathcal{P}_{p}^{i}(q)$ be one of its tiles with base figure $\mathcal{P}$ centered at the origin with vertices $G_{1} G_{2} \cdots G_{p}$ in the base plane of the model. Let $B_{K}^{\text {opt }}$ be the geodesic ball with center at the origin $K$ that touches the side surfaces of the infinite regular prism $\mathcal{P}_{p}^{i}(q)$. The radius of the ball $B_{K}^{\text {opt }}$ is denoted by $\rho^{\text {opt }}(K)$. Moreover, we define the regular prism $\mathcal{P}_{p}^{\text {opt }}(q)=A_{1} A_{2} \cdots A_{p} B_{1} B_{2} \cdots B_{p}$ with base figure $\mathcal{P}$ and with cover faces $A_{1} A_{2} \cdots A_{p}$ and $B_{1} B_{2} \cdots B_{p}$ touching $B_{K}^{\text {opt }}$. It is clear that the height $h_{p}^{\text {opt }}(q)$ of $\mathcal{P}_{p}^{\text {opt }}(q)$ is $2 \rho^{\text {opt }}(K)$.

The images of $\mathcal{P}_{p}^{\text {opt }}(q)$ by the fibre translations $\langle\tau\rangle$ where $h_{p}^{\text {opt }}(q)=$ $|\tau|=2 \rho^{\mathrm{opt}}(K)$ covers the infinite regular $\operatorname{prism} \mathcal{P}_{p}^{i}(q)$ and by the structure of the infinite prism tilings follows the rotations through $\omega=2 \pi / q$ about the fibre lines. $f_{i}$ maps the corresponding side face onto the neighboring one and thus the images of $\mathcal{P}_{p}^{\text {opt }}(q)$ fill the $\widetilde{\mathbf{S L}_{2} \mathbf{R}}$ space without overlap. These tilings are denoted by $\mathcal{T}_{p}^{n}(q)$. 
The height $h_{p}^{\mathrm{opt}}(q)$ of the prism $\mathcal{P}_{p}^{\mathrm{opt}}(q)$ is not equal to $\pi-2 \pi / p-2 \pi / q$ so the corresponding regular prism tiling is non-periodic. We note here that there are infinitely many non-periodic prism tilings derived from $\mathcal{T}_{p}^{n}(q)$.

For the density of the packing it is sufficient to relate the volume of the optimal ball to that of the solid $\mathcal{P}_{p}^{\text {opt }}(q)$. The density of the optimal ball packing of the prism tiling $\mathcal{T}_{p}^{n}(q)$ (with integer parameters $p \geq 3$ and $q>2 p /(p-2))$ can be computed by the formula:

$$
\delta_{p}^{\mathrm{opt}}(q):=\frac{\operatorname{Vol}\left(B_{K}^{\mathrm{opt}}\right)}{\operatorname{Vol}\left(\mathcal{P}_{p}^{\mathrm{opt}}(q)\right)} .
$$

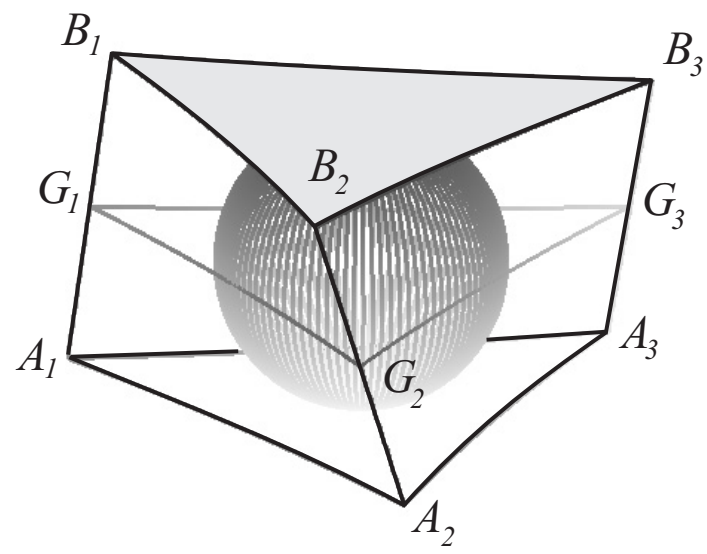

FIGURE 4 . The optimal prism $A_{1} A_{2} A_{3} B_{1} B_{2} B_{3}$ with optimal sphere for parameters $p=3, q=7$, of maximum radius $\rho^{\text {opt }}(K)$

In order to determine the optimal radius $\rho^{\mathrm{opt}}(K)$ we will use the following lemmas.

Lemma 2.2. The parametric equation of the side curve $c_{G_{1} G_{2}}$ of the base figure $\mathcal{P}$ is

$$
c_{p}^{q}(t)=\left(0, \sqrt{\sin \left(\frac{2 \pi}{p}+\frac{2 \pi}{q}\right)}\left(t \cos \left(\frac{2 \pi}{p}\right) \sin ^{2}\left(\frac{\pi}{p}+\frac{\pi}{q}\right)\right.\right.
$$




$$
\begin{aligned}
& -\frac{t}{2} \sin \left(\frac{2 \pi}{p}\right) \sin \left(\frac{2 \pi}{p}+\frac{2 \pi}{q}\right)+\sin ^{2}\left(\frac{\pi}{p}+\frac{\pi}{q}\right)(1-t) \\
& \left.+t^{2} \cos \left(\frac{\pi}{p}+\frac{\pi}{q}\right) \cos \left(\frac{\pi}{p}-\frac{\pi}{q}\right)\right) / \\
& \cdot\left(\sqrt{\left(\sin \left(\frac{2 \pi}{p}\right)+\sin \left(\frac{2 \pi}{q}\right)\right)}\right. \\
& \left.\cdot\left(\sin ^{2}\left(\frac{\pi}{p}+\frac{\pi}{q}\right)+t^{2} \cos ^{2}\left(\frac{\pi}{p}+\frac{\pi}{q}\right)\right)\right) \text {, } \\
& \sqrt[t]{\sin \left(\frac{2 \pi}{p}+\frac{2 \pi}{q}\right)}\left(\sin \left(\frac{2 \pi}{p}\right) \sin ^{2}\left(\frac{\pi}{p}+\frac{\pi}{q}\right)\right. \\
& +\frac{1}{2} \cos \left(\frac{2 \pi}{p}\right) \sin \left(\frac{2 \pi}{p}+\frac{2 \pi}{q}\right)(1-t) \\
& +\cos \left(\frac{\pi}{p}+\frac{\pi}{q}\right)\left(t \sin \left(\frac{2 \pi}{p}\right) \cos \left(\frac{\pi}{p}+\frac{\pi}{q}\right)\right. \\
& \left.\left.+\sin \left(\frac{\pi}{p}+\frac{\pi}{q}\right)(t-1)\right)\right) / \\
& \cdot\left(\sqrt{\left(\sin \left(\frac{2 \pi}{p}\right)+\sin \left(\frac{2 \pi}{q}\right)\right)}\right. \\
& \left.\cdot\left(\sin ^{2}\left(\frac{\pi}{p}+\frac{\pi}{q}\right)+t^{2} \cos ^{2}\left(\frac{\pi}{p}+\frac{\pi}{q}\right)\right)\right), \quad t \in[0,1] .
\end{aligned}
$$

The equation of the side curve $c_{G_{1} G_{2}}$ is derived by formulas (1.3) and (1.8). Therefore, they are congruent and their curvatures are equal in the $\widehat{\mathbf{S L}_{2} \mathbf{R}}$ sense. Moreover, the side curves are also congruent in the Euclidean sense; therefore, their curvatures are equal in the Euclidean sense as well. Our next lemma is obtained by applying some routine techniques commonly used in differential geometry.

Lemma 2.3. The curvature $C_{p}(q)$ of the side curves $c_{G_{i} G_{i+1}}, i=$ $1, \ldots, p, G_{p+1} \equiv G_{1}$, in the Euclidean sense is

$$
C_{p}(q)=\sqrt{\frac{\cos (\pi / p+\pi / q)(\sin (2 \pi / p)+\sin (2 \pi / q))}{\sin (\pi / p+\pi / q)(1-\cos (2 \pi / p))}} ;
$$


therefore, the side curves $c_{G_{i} G_{i+1}}\left(i=1, \ldots, p, G_{p+1} \equiv G_{1}\right)$ are Euclidean circular arcs of radius $r_{p}^{q}=1 / C_{p}(q)$.

\section{Remark 2.4.}

(i) It is easy to see that the asymptotic behavior of $C_{p}(q)$ is as follows:

$$
\lim _{q \rightarrow \infty}\left(C_{p}(q)\right)=\cot \left(\frac{\pi}{p}\right), \quad \lim _{p \rightarrow \infty}\left(C_{p}(q)\right)=\infty .
$$

(ii) Given a line and a point not on it, let $x$ be the distance from the point to the line along the perpendicular segment dropped from the point to the line. Let $\phi=\Pi(x)$ be the least angle, such that the line drawn through the point at that angle does not intersect the given line. This angle is called the angle of parallelism. By the famous formula of J. Bolyai it follows that $\log (\cot (\phi))=x$. Therefore, if we denote the distance of parallelism of the angle $\phi$ by $\Lambda(\phi)$, then

$$
\log \left(\lim _{q \rightarrow \infty}\left(C_{p}(q)\right)\right)=\log \left(\cot \left(\frac{\pi}{p}\right)\right)=\Lambda\left(\frac{\pi}{p}\right) .
$$

Table 1 lists the radii of curvature $r_{3}^{q}$ of the side curve $c_{G_{1} G_{2}}$ of the base figure $\mathcal{P}$.

\begin{tabular}{|c|c|c|c|c|}
\hline \multicolumn{5}{|c|}{ Table 1 } \\
\hline \hline$(p, q)$ & $(3,7)$ & $(3,8)$ & $(3,10)$ & $(3,1000)$ \\
\hline$C_{p}(q)$ & 0.286926 & 0.371579 & 0.453885 & 0.577339 \\
\hline$r_{p}^{q}$ & 3.485219 & 2.691215 & 2.203203 & 1.732085 \\
\hline
\end{tabular}

The maximum radius $\rho^{\mathrm{opt}}(K)$ of the balls $B_{K}^{\mathrm{opt}}$ can be determined by applying the above lemmas for all possible parameters as the distance between the origin and $c_{G_{1} G_{2}}$. The volumes $\operatorname{Vol}\left(B_{K}^{\mathrm{opt}}\right)$ can be computed by Theorem 1.8, and the volumes of the prisms $\mathcal{P}_{p}^{\text {opt }}(q)$ can be determined by Theorem 1.9 .

The above locally dense geodesic ball packings can be determined for all regular prism tilings $\mathcal{T}_{p}^{n}(q)(p, q$ as above); our results are summarized in Tables 2 and 3. 


\section{Remark 2.5.}

(i) The best density that we found is approximately 0.626606 , attained at parameters $p=29, q=3$, that is greater than the maximum density of the corresponding periodic geodesic ball packings under the groups $\mathbf{p q} \mathbf{2}_{\mathbf{1}}$.

(ii) The problems of finding the densest geodesic and translation ball packings are timely and intensively investigated in other Thurston geometries, as well (see e.g., $[4,10,11,12,13]$ ).

\begin{tabular}{|c|c|c|c|c|}
\hline \multicolumn{5}{|c|}{ Table 2.} \\
\hline$(p, q)$ & $\rho^{\mathrm{opt}}(K)$ & $\operatorname{Vol}\left(B_{K}^{\mathrm{opt}}\right)$ & $\operatorname{Vol}\left(\mathcal{P}_{p}^{\text {opt }}(q)\right)$ & $\delta_{p}^{\mathrm{opt}}(q)$ \\
\hline$(3,7)$ & 0.141564 & 0.011963 & 0.031767 & 0.376592 \\
\hline$(3,8)$ & 0.181760 & 0.025431 & 0.071377 & 0.356287 \\
\hline$(3,10)$ & 0.219795 & 0.045198 & 0.138101 & 0.327281 \\
\hline$(3,1000)$ & 0.274648 & 0.088981 & 0.428828 & 0.207499 \\
\hline$\vdots$ & $\vdots$ & $\vdots$ & $\vdots$ & $\vdots$ \\
\hline$(4,5)$ & 0.265319 & 0.080085 & 0.166705 & 0.480397 \\
\hline$(4,6)$ & 0.329239 & 0.154965 & 0.344779 & 0.449464 \\
\hline$(4,10)$ & 0.404230 & 0.292043 & 0.761956 & 0.383280 \\
\hline$(4,1000)$ & 0.440683 & 0.382228 & 1.378910 & 0.277196 \\
\hline$\vdots$ & $\vdots$ & $\vdots$ & : & : \\
\hline$(5,4)$ & 0.313435 & 0.133256 & 0.246171 & 0.541312 \\
\hline$(5,5)$ & 0.421241 & 0.332010 & 0.661684 & 0.501765 \\
\hline$(5,10)$ & 0.530638 & 0.686600 & 1.667047 & 0.411866 \\
\hline$(5,1000)$ & 0.562086 & 0.825191 & 2.639937 & 0.312580 \\
\hline$\vdots$ & : & : & : & : \\
\hline$(6,4)$ & 0.440687 & 0.382237 & 0.692229 & 0.552183 \\
\hline$(6,5)$ & 0.530638 & 0.686600 & 1.333638 & 0.514833 \\
\hline$(6,10)$ & 0.629251 & 1.188024 & 2.767592 & 0.429263 \\
\hline$(6,1000)$ & 0.658476 & 1.377893 & 4.124915 & 0.334042 \\
\hline$\vdots$ & $\vdots$ & $\vdots$ & : & : \\
\hline$(7,3)$ & 0.272637 & 0.087010 & 0.142753 & 0.609513 \\
\hline$(7,4)$ & 0.535202 & 0.705586 & 1.261041 & 0.559527 \\
\hline$(7,5)$ & 0.617496 & 1.117400 & 2.133913 & 0.523639 \\
\hline$(7,10)$ & 0.710652 & 1.772033 & 4.018646 & 0.440953 \\
\hline
\end{tabular}




\begin{tabular}{|c|c|c|c|c|}
\hline \multicolumn{5}{|c|}{ Table 2 (Continued). } \\
\hline \hline$(p, q)$ & $\rho^{\mathrm{opt}}(K)$ & $\operatorname{Vol}\left(B_{K}^{\mathrm{opt}}\right)$ & $\operatorname{Vol}\left(\mathcal{P}_{p}^{\mathrm{opt}}(q)\right)$ & $\delta_{p}^{\mathrm{opt}}(q)$ \\
\hline$(7,1000)$ & 0.738668 & 2.015812 & 5.785244 & 0.348440 \\
\hline$\vdots$ & $\vdots$ & $\vdots$ & $\vdots$ & $\vdots$ \\
\hline$(8,3)$ & 0.382143 & 0.245334 & 0.400179 & 0.613062 \\
\hline$(8,4)$ & 0.612113 & 1.086117 & 1.923010 & 0.564800 \\
\hline$(8,5)$ & 0.690221 & 1.608804 & 3.035751 & 0.529953 \\
\hline$(8,10)$ & 0.780165 & 2.422804 & 5.392115 & 0.449324 \\
\hline$(8,1000)$ & 0.807443 & 2.722797 & 7.589676 & 0.358750 \\
\hline$\vdots$ & $\vdots$ & $\vdots$ & $\vdots$ & $\vdots$ \\
\hline
\end{tabular}

\begin{tabular}{|c|c|c|c|c|}
\hline \multicolumn{5}{|c|}{ Table 3.} \\
\hline \hline$(p, q)$ & $\rho^{\text {opt }}(K)$ & $\operatorname{Vol}\left(B_{K}^{\text {opt }}\right)$ & $\left.\operatorname{Vol}_{\left(\mathcal{P}_{p}^{\text {opt }}\right.}(q)\right)$ & $\delta_{p}^{\text {opt }}(q)$ \\
\hline$(10,3)$ & 0.530638 & 0.686600 & 1.111365 & 0.617799 \\
\hline$\vdots$ & $\vdots$ & $\vdots$ & $\vdots$ & $\vdots$ \\
\hline$(20,3)$ & 0.914848 & 4.195479 & 6.706186 & 0.625613 \\
\hline$(20,4)$ & 1.094612 & 8.023914 & 13.755306 & 0.583332 \\
\hline$(20,5)$ & 1.163424 & 10.092704 & 18.275027 & 0.552268 \\
\hline$(20,10)$ & 1.245625 & 13.132701 & 27.392724 & 0.479423 \\
\hline$(20,1000)$ & 1.271043 & 14.216772 & 35.858024 & 0.396474 \\
\hline$\vdots$ & $\vdots$ & $\vdots$ & $\vdots$ & $\vdots$ \\
\hline$(28,3)$ & 1.088398 & 7.855861 & 12.537440 & 0.626592 \\
\hline$(\mathbf{2 9 , 3})$ & $\mathbf{1 . 1 0 6 3 1 1}$ & $\mathbf{8 . 3 4 8 3 1 0}$ & $\mathbf{1 3 . 3 2 3 0 5 4}$ & $\mathbf{0 . 6 2 6 6 0 6}$ \\
\hline$(30,3)$ & 1.123593 & 8.847342 & 14.119487 & 0.626605 \\
\hline$\vdots$ & $\vdots$ & $\vdots$ & $\vdots$ & $\vdots$ \\
\hline$(35,3)$ & 1.201914 & 11.432334 & 18.250297 & 0.626419 \\
\hline$\vdots$ & $\vdots$ & $\vdots$ & $\vdots$ & $\vdots$ \\
\hline$(40,3)$ & 1.269482 & 14.148085 & 22.599777 & 0.626028 \\
\hline$\vdots$ & $\vdots$ & $\vdots$ & $\vdots$ & $\vdots$ \\
\hline$(52,3)$ & 1.401728 & 21.089811 & 33.761388 & 0.624673 \\
\hline$\vdots$ & $\vdots$ & $\vdots$ & $\vdots$ & $\vdots$ \\
\hline$(72,3)$ & 1.565173 & 33.642710 & 54.088487 & 0.621994 \\
\hline & & & & \\
\hline
\end{tabular}




\section{REFERENCES}

1. K. Böröczky and A. Florian, Uber die dichteste Kugelpackung im hyperbolischen Raum, Acta Math. Hung. 15 (1964), 237-245.

2. E. Molnár, The projective interpretation of the eight 3-dimensional homogeneous geometries, Beitr. Alg. Geom. 38 (1997), 261-288.

3. E. Molnár and J. Szirmai, Symmetries in the 8 homogeneous 3-geometries, Symmetry Cult. Sci. 21 (2010), 87-117.

4. _ Classification of Sol lattices, Geom. Ded. 161 (2012), 251-275.

5. Volumes and geodesic ball packings to the regular prism tilings in $\widetilde{\mathbf{S L}_{2} \mathbf{R}}$ space, Publ. Math. Debr. 84 (2014), 189-203.

6. E. Molnár, J. Szirmai and A. Vesnin, Projective metric realizations of conemanifolds with singularities along 2-bridge knots and links, J. Geom. 95 (2009), 91-133.

7. _ Packings by translation balls in $\widetilde{\mathbf{S L}_{2} \mathbf{R}}$, J. Geom. 105 (2014), 287306.

8. J.G. Ratcliffe, Foundations of hyperbolic manifolds, 2nd ed, Grad. Texts Math. 149, Springer, New York, 2006.

9. P. Scott, The geometries of 3-manifolds, Bull. Lond. Math. Soc. 15 (1983), 401-487.

10. J. Szirmai, The densest geodesic ball packing by a type of Nil lattices, Beitr. Alg. Geom. 48 (2007), 383-398.

11. Geodesic ball packing in $\mathbf{S}^{2} \times \mathbf{R}$ space for generalized Coxeter space groups, Beitr. Alg Geom. 52 (2011), 413-430.

12. Geodesic ball packing in $\mathbf{H}^{2} \times \mathbf{R}$ space for generalized Coxeter space groups, Math. Comm. 17 (2012), 151-170.

13. Lattice-like translation ball packings in Nil space, Publ. Math. Debrecen 80 (2012), 427-440.

14. , Regular prism tilings in $\widehat{\mathbf{S L}_{2} \mathbf{R}}$ space, Aequat. Math. 88 (2014), $67-79$.

15. A candidate to the densest packing with equal balls in the Thurston geometries, Beitr. Alg. Geom. (2013).

16. W.P. Thurston and S. Levy, eds., Three-dimensional geometry and topology, Princeton University Press, Princeton, 1997.

Budapest University of Technology and Economics Institute of Mathematics, Department of Geometry, P.O. Box 91, Budapest H-1521, Hungary Email address: szirmai@math.bme.hu 